\title{
Factores que afectan la preñez en vacas Brahman sometidas a inseminación artificial a tiempo fijo
}

\author{
Factors affecting pregnancy in Brahman cows submitted to \\ fixed-time artificial insemination
}

\author{
Adriana Correa-Orozco, ${ }^{1 *}$ M.Sc, Luis Uribe-Velásquez, ${ }^{2}$ Ph.D, Erik Pulgarín-Velásquez, ${ }^{1}$ MVZ. \\ ${ }^{1}$ Universidad de Caldas, Facultad de Ciencias Agropecuarias, Grupo de Investigación CIENVET. \\ Manizales, Colombia. ${ }^{2}$ Universidad de Caldas, Facultad de Ciencias Agropecuarias, Departamento de \\ Salud Animal. Manizales, Colombia. *Correspondencia: adrianacorreao@hotmail.com
}

Recibido: Agosto de 2011; Aceptado: Enero de 2012.

\section{RESUMEN}

Objetivo. Evaluar la relación entre la condición corporal (CC), la manifestación de estro, las concentraciones plasmáticas de estradiol, el diámetro del folículo mayor y la preñez, en vacas Bos indicus amamantando, sometidas a un protocolo de inseminación artificial a tiempo fijo (IATF). Materiales y métodos. Treinta y ocho vacas Brahman recibieron un dispositivo intravaginal de progesterona + benzoato de estradiol en el día 0; retiro del dispositivo + dinoprost + gonadotropina coriónica equina en el día 9 y la IATF en el día 12. La preñez fue diagnosticada 90 días después de la IATF. Resultados. No hubo efecto de la CC sobre la manifestación de estro, las concentraciones plasmáticas de estradiol o el diámetro del folículo mayor $(p>0.05)$. El diámetro del folículo mayor no influenció las concentraciones plasmáticas de estradiol $(p>0.05)$. El diámetro del folículo mayor y las concentraciones plasmáticas de estradiol no influenciaron la manifestación de estro $(p>0.05)$. La tasa de preñez por IATF no fue afectada ( $p>0.05$ ) por la $C C$, la manifestación de estro o las concentraciones plasmáticas de estradiol. Hubo una tendencia $(p=0.10)$ de una relación directa entre el diámetro del folículo mayor y la tasa de preñez por IATF. Conclusiones. La CC, las concentraciones plasmáticas de estradiol y la manifestación de estro no influenciaron la tasa de preñez por IATF en vacas Brahman sometidas a un protocolo de sincronización basado en progesterona. Sin embargo, el diámetro del folículo mayor presentó tendencia al impacto positivo sobre la tasa de preñez por IATF.

Palabras clave: Condición corporal, estradiol, folículos ováricos, ganado de carne (Fuente: Agrovoc). 


\section{ABSTRACT}

Objective. To examine the relationship between body condition score (BCS), the occurrence of estrous, estradiol plasma concentrations, diameter of largest ovarian follicle (LF) and pregnancy rate in suckled Bos indicus cows submitted to a fixed-time artificial insemination (FTAI) protocol. Material and methods. Thirty eight Brahman cows received an intravaginal device of progesterone + estradiol benzoate on Day 0, device withdrawal + dinoprost + equine chorionic gonadotropin on Day 9, and FTAI on Day 12. Pregnancy was diagnosed 90 days after FTAI. Results. There was no effect of BCS on the occurrence of estrous, estradiol plasma concentrations or diameter of the LF $(p>0.05)$. The diameter of LF did not influence ( $p>0.05)$ estradiol plasma concentrations. The diameter of the LF and estradiol plasma concentrations did not influence the occurrence of estrous $(p>0.05)$. Pregnancy rate per FTAI was not affected $(p>0.05)$ by BCS, the occurrence of estrous or plasma concentrations of estradiol. There was a tendency $(p=0.10)$ of a direct relationship between diameter of the LF and pregnancy rate FTAI. Conclusions. BCS, plasma concentrations of estradiol and occurrence of estrous did not influence pregnancy rate per FTAI in Brahman cows submitted to progesterone-based protocol. However, the diameter of LF had a tendency to positive impact on pregnancy rate per FTAI.

Key words: Beef cattle, body condition, oestradiol, ovarian follicles (Source: Agrovoc).

\section{INTRODUCCIÓN}

Existen diferencias en la dinámica folicular de las hembras Bos taurus y Bos indicus, lo que sugiere la importancia de conocer las particularidades del desarrollo folicular en las especies bovinas, con el fin de implementar protocolos de manejo reproductivo distintos para mejorar la eficiencia reproductiva (1). Según Baruselli et al (2), se sugiere que los tratamientos con dispositivos liberadores de progesterona $\left(P_{4}\right)$ y benzoato de estradiol, pueden mejorar el desempeño reproductivo en vacas Bos indicus con cría, debido a su efecto favorable sobre la frecuencia pulsátil de la hormona luteinizante, el crecimiento del folículo y la ovulación. Además, el uso de la gonadotropina coriónica equina (eCG) al momento del retiro del dispositivo de $\mathrm{P}_{4}$ en un programa de inseminación artificial a tiempo fijo (IATF) mejora la tasa de preñez (2-4). Sin embargo, la variabilidad en la respuesta a los tratamientos hormonales, el tiempo requerido para los mismos y la detección del estro, particularmente en ganado Bos indicus, limitan la aplicación y el éxito de esas tecnologías (5).

La ovulación de folículos pequeños produce baja fertilidad debido posiblemente, al desarrollo de un cuerpo lúteo de tamaño menor, alterando las concentraciones circulantes de $\mathrm{P}_{4}(6)$. Además, de aumentar la tasa ovulación, otros factores tales como la producción de estradiol $\left(E_{2}\right)$ endógeno, la competencia del oocito, el diámetro del cuerpo lúteo y la concentración de $\mathrm{P}_{4}$ en el ciclo estral siguiente, también resultan de la ovulación de un folículo más grande, el cual finalmente puede beneficiar la fertilidad en vacas de carne después de un protocolo de IATF (7).
En Colombia, para el año 2005 se estimó un hato de 23 millones de bovinos, de los cuales el $72 \%$ corresponde a ganado Bos indicus (8). Debido a la predominancia de este tipo de ganado en el país, es importante contribuir al desarrollo e investigación en explotaciones dedicadas a la cría de ganado de carne con razas Bos indicus. El desafío más importante para este tipo de animales en climas tropicales, es el anestro posparto prolongado; por lo tanto, los tratamientos que mejoren esta condición, tienen impactos positivos sobre las ganaderías de carne (9). El objetivo del presente trabajo fue evaluar la relación existente entre la condición corporal (CC), la manifestación de estro, el diámetro del folículo mayor, las concentraciones plasmáticas de $E_{2}$ y la tasa de preñez, en vacas Bos indicus sometidas a un protocolo de IATF.

\section{MATERIALES Y MÉTODOS}

Localización. El experimento se realizó entre el mes de junio y el mes de octubre de 2010 en la Hacienda La Indiana, ubicada en el municipio de Buenavista, Departamento de Córdoba, Colombia $\left(8^{\circ} 03^{\prime} 25.40^{\prime \prime} \mathrm{N} / 75^{\circ} 29^{\prime} 19.95^{\prime \prime} \mathrm{O}, 68\right.$ $\mathrm{msnm}$ ). El clima se caracteriza por ser cálido húmedo según la clasificación de Holdridge, con una precipitación anual entre 2000 a 2500 mm, temperatura media de $27^{\circ} \mathrm{C}$ y humedad relativa entre $80-85 \%(10)$.

Animales. Se utilizaron 38 vacas Brahman (Bos indicus) en amamantamiento, normales al chequeo reproductivo transrectal (cuatro de primer parto y 34 multíparas) y con $126.6 \pm 4.4$ 
días de posparto. Las vacas fueron mantenidas en pasturas de Brachiaria decumbens, Brachiaria humidicola y Brachiaria dictyoneura, con libre acceso al agua y sal mineralizada ( $8 \%$ fósforo).

La CC fue evaluada el primer día del protocolo de sincronización del estro, utilizando una escala de 1 a 5 ( $1=$ emaciada, $5=$ obesa) (11). También se registró el peso corporal (PC) de cada animal. La CC fue categorizada como $\leq 3.25$ ó $>3.25$, con el fin de analizar su relación con el diámetro del folículo mayor, las concentraciones de estradiol, la manifestación del estro y con la tasa preñez por IATF.

Protocolo de sincronización del estro. Independiente del día del ciclo estral (inicio del tratamiento $=$ día 0 ), las vacas recibieron un dispositivo intravaginal que contenía $1.3 \mathrm{~g}$ de progesterona (CIDR ${ }^{\circledR}$, Pfizer Inc, New Zealand) y una inyección intramuscular (im) de $2.5 \mathrm{mg}$ de benzoato de estradiol (BE; $1.0 \mathrm{ml}$ Estro-Zoo ${ }^{\circledR}$, Laboratorios Zoo Ltda, Bogotá, Colombia). Al día 9, el dispositivo intravaginal fue retirado y las hembras fueron tratadas con $25 \mathrm{mg}$ de dinoprost como trometamina im (PG; $5.0 \mathrm{ml}$ Lutalyse ${ }^{\circledR}$, Pfizer Inc, Kalamazoo, USA) y 400 UI de gonadotropina coriónica equina im (eCG; 2.0 $\mathrm{ml}$ Folligon ${ }^{\circledR}$, Intervet International, Booxmeer, Holanda). Las vacas fueron inseminadas con semen congelado de fertilidad probada, 56 a 60 horas después de retirar el dispositivo de progesterona (Figura 1). Además, 20 días después de la IATF, dos grupos de vacas $(n=18$ y $n=20$ ), estuvieron cada uno en pastoreo con un toro de raza Brahman para monta natural.

Detección del estro. El estro fue determinado en un subgrupo de vacas $(n=20)$ mediante un adhesivo (Estrotect, IVP, Spring Valley, Wiscosin, USA), el cual fue colocado entre la cadera y la base de la cola el día del retiro del CIDR. La manifestación de estro se estableció el día de la IATF por la activación de cada adhesivo.

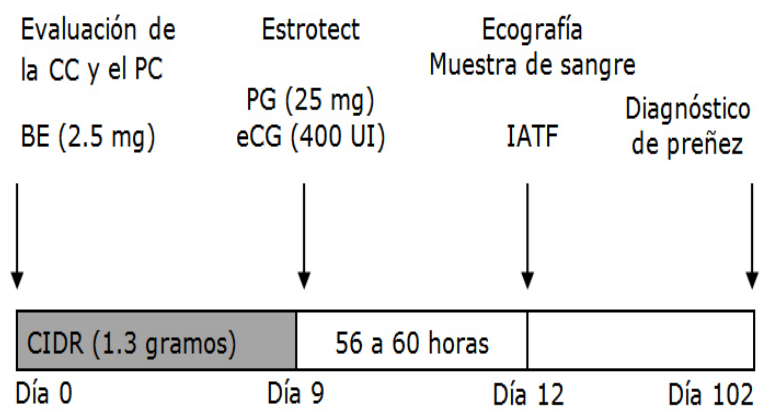

Figura 1. Protocolo de sincronización del estro y actividades realizadas durante el experimento.
Ecografía ovárica y clases de folículos. Los ovarios fueron examinados por ecografía transrectal $(6 / 8 \mathrm{MHz}$, Aquila Pro, Esaote Europe B.V., Maastricht, The Netherlands), con el objetivo de medir el diámetro del folículo mayor en el momento de la IATF. Los folículos fueron clasificados en cuatro categorías basadas en la media y la desviación estándar (DE) del diámetro (7). Por lo tanto, los folículos fueron categorizados como $<8.0 \mathrm{~mm}$; 8.1 a $10.9 \mathrm{~mm}$; 11.0 a $16.7 \mathrm{~mm}$ y $>16.7 \mathrm{~mm}$.

\section{Determinación de las concentraciones plasmáticas de estradiol por} radioinmunoanálisis. Se tomó una muestra de sangre de cada vaca por venopunción coccígea en tubo Vacutainer ${ }^{\circledR}$ con EDTA el día de la IATF. El plasma se obtuvo por centrifugación $(1178 \times \mathrm{g}$ por $5 \mathrm{~min}$ ) y fue almacenado en microtubos a $-20^{\circ} \mathrm{C}$. Las concentraciones plasmáticas de $17 \beta$-estradiol fueron determinadas por radioinmunoanálisis en fase sólida utilizando el kit comercial ImmuChem ${ }^{\mathrm{TM}}$ Coated Tub $17 \beta$-Estradiol ${ }^{\circledR}$ (MP Biomedicals, Costa Mesa, CA, USA), en el Laboratorio de Radioinmunoanálisis de la Universidad Nacional de Colombia, Sede Bogotá. Cada muestra fue medida en duplicado y la media fue usada como el valor. Todas las muestras fueron incluidas en un sólo ensayo y se obtuvo un coeficiente de variación intra-ensayo de $15.7 \%$ y una sensibilidad de $0.3 \mathrm{pg} / \mathrm{ml}$.

Diagnóstico de preñez. Se realizó por palpación transrectal 90 días después de la IATF y se registró el tiempo de la preñez. Se consideró que las hembras con 90 días de gestación concibieron en la IATF y aquellas que tenían 70 días concibieron en la monta natural. La tasa de preñez por IATF fue calculada como la proporción de vacas preñadas dividida por el número de vacas inseminadas. La tasa de preñez por monta natural, que fue definida como el número de vacas preñadas por monta natural dividido entre el número de vacas sometidas a monta natural.

Diseño experimental y análisis estadístico. Se realizó un diseño de estimación de parámetros, con los ajustes pertinentes para el efecto de bloque, en el cual las vacas fueron bloqueadas de acuerdo al día de inicio del protocolo de sincronización del estro (Grupo $1, \mathrm{n}=18$; Grupo 2, $\mathrm{n}=20$ ).

Mediante análisis de varianza se evalúo: (a) el efecto de la categoría de CC; la categoría del diámetro del folículo mayor y las concentraciones plasmáticas de estradiol sobre la manifestación de estro (variable binaria); (b) el efecto de la categoría del diámetro del folículo mayor y de la categoría de la CC sobre 
las concentraciones plasmáticas de estradiol y tasa de preñez por IATF; (c) el efecto de la manifestación de estro sobre la tasa de preñez por IATF.

Se determinaron los coeficientes de correlación de Pearson entre la condición corporal (CC), el peso corporal (PC), el diámetro del folículo mayor y las concentraciones plasmáticas de estradiol. Además, se determinó por análisis de regresión la relación entre el diámetro del folículo mayor y las concentraciones plasmáticas de estradiol.

La tasa de preñez por IATF (variable binaria) fue analizada mediante regresión logística, considerando como factores en el modelo la CC, el diámetro del folículo mayor y las concentraciones plasmáticas de estradiol. El modelo final sólo conservó el diámetro del folículo mayor, debido a que mostró una tendencia $(p=0.10)$. La curva de regresión logística para la tasa de preñez por IATF y diámetro del folículo mayor fue creada con el coeficiente suministrado por el análisis de datos del programa SAS (12) y la formula $\mathrm{P}_{\mathrm{i}}=\mathrm{e}^{(\mathrm{ni})} / 1+\mathrm{e}^{(\mathrm{ni})}$, en la cual la $\mathrm{p}_{\mathrm{i}}$ es la probabilidad estimada, la $\mathrm{n}$ es el intercepto y la i es el estimador del punto.

Los efectos de la CC y del PC al inicio del protocolo sobre la categoría del diámetro del folículo mayor también fueron determinados por regresión logística. Se obtuvo de cada variable incluida en el modelo estadístico el odds ratio (OR) y el intervalo de confianza (IC) al $95 \%$.

Se consideraron diferencias significativas cuando $p \leq 0.05$ y tendencias cuando $p \leq 0.10$. Los resultados se presentan como medias de mínimos cuadrados \pm error estándar (EE). Los análisis estadísticos fueron realizados con el programa SAS (12).

\section{RESULTADOS}

La media \pm EE y el intervalo de las variables estudiadas, se presentan en la tabla 1 . Se encontró una correlación positiva (0.60) y significativa $(p<0.0001)$ entre la CC y el PC (Tabla 2).

Manifestación de estro. En el subgrupo de vacas que fue determinado el estro mediante adhesivo $(n=20)$, sólo siete de ellas mostraron estro (35.0\%). La categoría de la CC (Tabla 3) y la categoría del diámetro del folículo mayor (Tabla 4), no afectaron ( $p>0.05$ ) la manifestación de estro. Tampoco se encontró asociación entre
Tabla 1. Media \pm error estándar (EE) y el intervalo de la condición corporal (CC), el peso corporal (PC), el diámetro del folículo mayor y las concentraciones plasmáticas de estradiol $\left(E_{2}\right)$, en vacas Brahman amamantando sometidas a un protocolo de IATF basado en progesterona/estradiol.

\begin{tabular}{lcc}
\hline Variable & Media \pm EE & Intervalo \\
\hline $\mathrm{CC}$ & $3.45 \pm 0.06$ & 2.5 a 4.0 \\
$\mathrm{PC}, \mathrm{kg}$ & $522.20 \pm 9.81$ & 357 a 648 \\
$\begin{array}{l}\text { Diámetro del folículo mayor, } \\
\mathrm{mm}\end{array}$ & $13.76 \pm 0.47$ & 8.30 a 19.10 \\
$\begin{array}{l}\text { Concentraciones plasmáticas } \\
\text { de } \mathrm{E}_{2}, \mathrm{pg} / \mathrm{ml}\end{array}$ & $23.99 \pm 0.96$ & 15.01 a 36.90 \\
\hline
\end{tabular}

Tabla 2. Correlaciones de Pearson entre la condición corporal (CC), el peso corporal (PC), el diámetro del folículo mayor y las concentraciones plasmáticas de estradiol $\left(E_{2}\right)$, en vacas Brahman amamantando sometidas a un protocolo de IATF basado en progesterona/estradiol.

\begin{tabular}{lcccc}
\hline \multicolumn{1}{c}{ Variable } & CC & PC & $\begin{array}{c}\text { Diámetro } \\
\text { del folículo } \\
\text { mayor }\end{array}$ & $\begin{array}{c}\text { Concentraciones } \\
\text { plasmáticas de } E_{2}\end{array}$ \\
\hline CC & & $0.60 *$ & -0.12 & 0.09 \\
PC & $0.60 *$ & & 0.11 & -0.18 \\
$\begin{array}{l}\text { Diámetro del } \\
\text { folículo mayor }\end{array}$ & -0.12 & 0.11 & & 0.09 \\
$\begin{array}{l}\text { Concentraciones } \\
\text { plasmáticas de } \mathrm{E}_{2}\end{array}$ & 0.09 & -0.18 & 0.09 & \\
\hline
\end{tabular}

$* \mathrm{p}<0.0001$

Tabla 3. Manifestación de estro, concentraciones plasmáticas de estradiol $\left(E_{2}\right)$ y tasa de preñez por IATF de acuerdo a la categoría de condición corporal (CC), en vacas Brahman amamantando sometidas a un protocolo de IATF basado en progesterona/ estradiol.

\begin{tabular}{lccc}
\hline \multicolumn{1}{c}{ Variable } & \multicolumn{2}{c}{ Categorías de CC } & P \\
\cline { 2 - 3 } & $\mathbf{5 3 . 2 5}$ & $\mathbf{3 . 2 5}$ & \\
\cline { 2 - 3 } Número de vacas & 14 & 24 & - \\
Manifestación de Estro, \% & 33.5 & 33.7 & 0.99 \\
$\begin{array}{l}\text { Concentraciones plasmáticas de } \\
\mathrm{E}_{2} \text { pg/ml }\end{array}$ & $22.25 \pm 1.77$ & $23.08 \pm 1.40$ & 0.70 \\
Tasa de preñez por IATF, \% & 19.8 & 11.9 & 0.52 \\
\hline
\end{tabular}

las concentraciones plasmáticas de $E_{2}$ y la manifestación de estro ( $p>0.05)$.

Diámetro del folículo mayor en el momento de la IATF. El diámetro del folículo mayor no presentó correlación ( $p>0.05)$ con la CC ni con el PC (Tabla 2). La categoría del folículo mayor tampoco se relacionó con la CC o el PC ( $p>0.05)$.

Concentraciones plasmáticas de estradiol $\left(E_{2}\right)$ en el momento de la IATF. No se demostró correlación $(p>0.05)$ de las concentraciones de 
$\mathrm{E}_{2}$ con la CC ni con el PC (Tabla 2). La categoría de la CC (Tabla 3 ) tampoco afectó esta variable $(p>0.05)$.

No se encontró asociación significativa entre el diámetro del folículo mayor y las concentraciones plasmáticas de $\mathrm{E}_{2}$ (Tabla 2), en el momento de la IATF $(p>0.05)$. La categoría del diámetro del folículo mayor (Tabla 4) no influenció las concentraciones plasmáticas de $\mathrm{E}_{2}(\mathrm{p}>0.05)$.

Tabla 4. Manifestación de estro, concentraciones plasmáticas de estradiol $\left(E_{2}\right)$ y tasa de preñez por IATF de acuerdo a la categoría del diámetro del folículo mayor en el momento de la IATF, en vacas Brahman amamantando sometidas a un protocolo de IATF basado en progesterona/estradiol.

\begin{tabular}{|c|c|c|c|c|}
\hline \multirow[t]{2}{*}{ Variable } & \multicolumn{3}{|c|}{$\begin{array}{c}\text { Categorías del diámetro del folículo } \\
\text { mayor }\end{array}$} & \multirow[t]{2}{*}{$\mathbf{p}$} \\
\hline & $\begin{array}{c}8.1 \text { a } 10.9 \\
\mathrm{~mm}\end{array}$ & $\begin{array}{c}11.0 \text { a } 16.7 \\
\mathrm{~mm}\end{array}$ & $\begin{array}{c}>16.7 \\
\mathbf{m m}\end{array}$ & \\
\hline Número de vacas & 7 & 25 & 6 & - \\
\hline $\begin{array}{l}\text { Manifestación de } \\
\text { Estro, \% }\end{array}$ & 0 & 49.7 & 50.8 & 0.16 \\
\hline $\begin{array}{l}\text { Concentraciones } \\
\text { plasmáticas de } E_{2} \text {, } \\
\mathrm{pg} / \mathrm{ml}\end{array}$ & $21.33 \pm 2.42$ & $25.18 \pm 1.21$ & $21.49 \pm 2.43$ & 0.19 \\
\hline $\begin{array}{l}\text { Tasa de preñez } \\
\text { por IATF, \% }\end{array}$ & 0 & 13.5 & 34.8 & 0.23 \\
\hline
\end{tabular}

Tasa de preñez por IATF. La tasa de preñez por IATF fue de $13.2 \%(5 / 38)$. No se encontró asociación $(p>0.05)$ entre la CC y la tasa de preñez por IATF y en el análisis por categoría de CC (Tabla 3), tampoco se estableció efecto de esta variable sobre la tasa de preñez por IATF $(p>0.05)$. No se halló efecto de la manifestación de estro sobre la tasa de preñez por IATF ( $p>0.05)$, con valores de $7.7 \%(1 / 13)$ para vacas que no mostraron estro y $28.6 \%(2 / 7)$ para las vacas detectadas en estro.

Se determinó una tendencia de relación positiva entre el diámetro del folículo mayor y la tasa de preñez por IATF $(p=0.10 ; O R=1.450 ;$ IC $=0.933$ a 2.252), en la cual el aumento en el diámetro del folículo mayor en el momento de la IATF incrementa la probabilidad de preñez por IATF (Figura 2).

Es interesante que, en el análisis adicional por categoría del diámetro del folículo mayor (Tabla $4)$, no se evidenció el efecto de esta variable sobre la tasa de preñez por IATF $(p>0.05)$.

No se halló relación $(p>0.05)$ entre las concentraciones plasmáticas de $\mathrm{E}_{2}$ y la tasa de preñez por IATF.

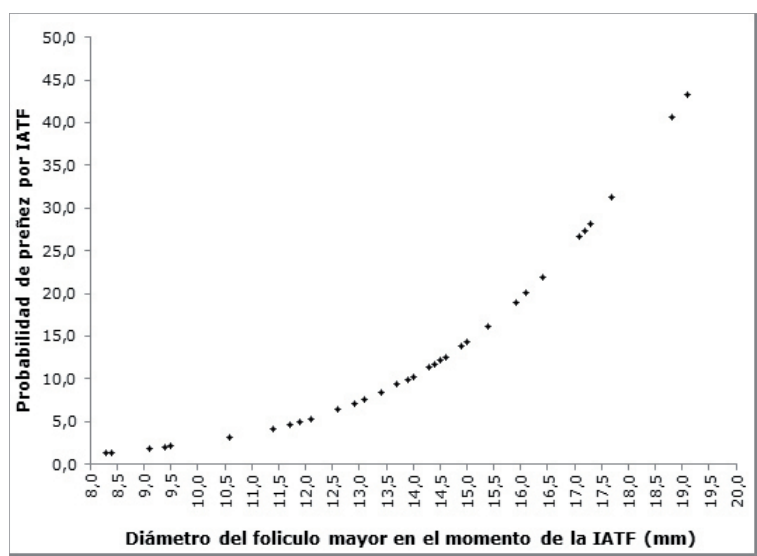

Figura 2. Probabilidad de preñez por IATF en vacas Brahman amamantando $(n=38)$, según el diámetro del folículo mayor en el momento de la IATF [probabilidad de preñez por IATF $=e(-7.3648+0.3715$ $x$ el diámetro del folículo mayor en el momento de la IATF $) / 1+$ e(-7.3648 + $0.3715 \times$ el diámetro del folículo mayor en el momento de la IATF); $\mathrm{p}=0.10$ )].

Tasa de preñez por monta natural. La tasa de preñez por monta natural después del protocolo de IATF (20 días) fue de $36.8 \%$ (14/38).

\section{DISCUSIÓn}

En esta investigación, en el subgrupo de vacas que fue determinado el estro, la categoría de la CC no afectó la manifestación de estro. De forma contraria, en otros estudios, a medida que se aumentaba la CC, se incrementaba el porcentaje de vacas inseminadas a estro detectado (13) y la manifestación de estro en vacas sometidas a IATF (7).

La categoría del diámetro del folículo mayor tampoco afectó la manifestación de estro en este experimento. De forma diferente, en hembras Angus $x$ Nelore en anestro (13) y en vacas Nelore y Nelore $x$ Angus en amamantamiento sincronizadas con $\mathrm{P}_{4} 0$ progestágenos (7), las vacas con folículos más grandes tuvieron una mayor probabilidad de mostrar estro.

En el presente estudio, las concentraciones plasmáticas de $\mathrm{E}_{2}$ no afectaron la manifestación de estro. Por el contrario, en vacas de carne cruzadas, sometidas a IATF con GnRH+PG+GnRH y a IA a estro detectado, las concentraciones de $E_{2}$ en suero fueron mayores en vacas detectadas en estro en comparación con aquellas no detectadas en estro (14). 
La ausencia de efecto de la categoría de la CC, de la categoría del diámetro del folículo mayor y de las concentraciones plasmáticas de $\mathrm{E}_{2}$ sobre la manifestación de estro en esta investigación, se puede deber a que, como consecuencia de la falta de correlación de la CC con las concentraciones de $E_{2}$, $y$ a su vez de ésta variable con el diámetro del folículo mayor y la tasa de preñez por IATF, fue probable la falta de asociación con la manifestación de estro, debido a que este comportamiento depende fisiológicamente en gran parte de las concentraciones de $\mathrm{E}_{2}$.

En la presente investigación, no se encontró relación del diámetro del folículo mayor en el momento de la IATF o de sus categorías, con la CC ni con el PC. Resultados similares fueron reportados en vacas Nelore sometidas a IATF (15). Contrariamente, otros autores encontraron que las vacas con mejor CC, tuvieron folículos más grandes $(13,16,17)$. No obstante, Meneghetti et al (9) obtuvieron resultados contradictorios en varios experimentos.

En el estudio de Sá Filho et al (18), el tratamiento con eCG provocó incrementos en la fertilidad debido, principalmente, al aumento en las tasas de preñez de vacas posparto no cíclicas o vacas con baja CC. Además, bajo esas circunstancias, el tratamiento con eCG al momento de retirar el progestágeno puede proporcionar un soporte gonadotrópico adicional, al aumentar la tasa de crecimiento del folículo. Ésto podría explicar en parte, la ausencia de efecto de la CC sobre el diámetro del folículo mayor en la presente investigación, en donde la eCG pudo haber aumentado el diámetro del folículo mayor en vacas con $\mathrm{CC} \leq 3.25$, de tal forma que aquellas hembras lograron alcanzar un diámetro folicular similar al de vacas con $\mathrm{CC}>3.25$.

En este experimento, las concentraciones plasmáticas de $\mathrm{E}_{2}$ en el momento de la IATF no se correlacionaron con el PC, la CC o sus categorías. Resultados similares fueron reportados en vacas Angus $x$ Hereford multíparas (16) y en novillas de carne Bos taurus (19). La falta de relación entre la $\mathrm{CC}$ y las concentraciones plasmáticas de $\mathrm{E}_{2}$ en el presente estudio fue, probablemente, una consecuencia directa de la ausencia de asociación entre la CC y el diámetro del folículo de mayor tamaño, debido a que las concentraciones de esta hormona dependen de la producción por parte del folículo.

En el presente estudio, no hubo relación entre el diámetro del folículo mayor y las concentraciones plasmáticas de $E_{2}$. De forma similar, en vacas Angus cruzadas multíparas inducidas a ovular con $G n R H+P G+G n R H$, el tamaño del folículo ovulatorio no influenció las concentraciones sanguíneas de $E_{2}$ (14). Contrario a esto, en novillas (19) y en vacas de carne $(20,21)$, las concentraciones de $E_{2}$ en sangre fueron correlacionadas positivamente con el tamaño del folículo dominante.

Una sola dosis de eCG es suficiente para estimular el crecimiento de múltiples folículos (15). En la presente investigación, la aplicación de eCG pudo haber estimulado el desarrollo de varios folículos ováricos en algunos animales, lo cual incrementó la secreción de $\mathrm{E}_{2}$ no sólo del folículo de mayor tamaño sino también de otros folículos en desarrollo, razón por la cual, probablemente, no se pudo correlacionar las concentraciones plasmáticas de $E_{2}$ con el diámetro del folículo de mayor en el momento de la IATF.

Con el fin de correlacionar las concentraciones endógenas de $\mathrm{E}_{2}$ con el tamaño del folículo mayor en el momento de la IATF, no se aplicó una segunda dosis de un éster de $E_{2}$ (BE o cipionato de estradiol) como inductor de la ovulación, lo cual posiblemente, puede afectar la tasa de preñez por IATF (13.2\%).

Según Sá Filho et al (22), es importante resaltar que la eficacia de los protocolos basados en $\mathrm{P}_{4}$ puede ser influenciada por la condición ovárica al momento de iniciar el protocolo. En las hembras que presentan concentraciones de $\mathrm{P}_{4}$ bajas en el momento de colocar los dispositivos de $P_{4}$, posiblemente el tratamiento con $P_{4} / E_{2}$ no induzca consistentemente la regresión del folículo dominante, con la posterior ovulación de un oocito viejo al momento de retirar el dispositivo, provocando reducidas tasas de preñez en la IATF (23). La variabilidad en el inicio de una nueva onda en los protocolos de sincronización, genera en los folículos que van a ser inducidos a ovular al final del programa de sincronización, variaciones en el tamaño, la madurez y la capacidad esteroidogénica (24). Los folículos con perfiles de crecimiento prolongados (folículos persistentes), y sus efectos negativos sobre las tasas de preñez, ya han sido descritos ampliamente en la literatura.

La condición reproductiva de las vacas (ciclicidad o anestro) y la sincronización de una nueva onda folicular al inicio del protocolo, con la posibilidad de dar origen a folículos persistentes, son factores que pudieron afectar la tasa de preñez por IATF obtenida en el presente experimento. Asimismo, existen fallas propias de los protocolos de IATF, en los cuales se ha reportado diferentes respuestas ováricas debidas al día del ciclo estral en el cual se inicia el protocolo de sincronización. 
En este experimento, la CC no afectó la tasa de preñez por IATF. Varios autores han reportado resultados similares en hembras bovinas tratadas con protocolos basados en $\mathrm{P}_{4} / \mathrm{E}_{2}(25,26,27)$. Sin embargo, Meneghetti et al (9) encontraron resultados contradictorios en vacas Bos indicus tratadas con un protocolo de IATF similar, en donde en tres de cuatro experimentos, no hubo asociación entre la CC y la tasa de preñez, y en el cuarto, la tasa de preñez fue afectada positivamente por la CC.

En un estudio realizado en Brasil, se concluyó que, la CC fue un factor de confianza para predecir la tasa de preñez en vacas Nelore sometidas a un protocolo de IATF basado en $\mathrm{P}_{4} / \mathrm{E}_{2} \mathrm{y}$ destete temporal (15). Asimismo, varias investigaciones realizadas en Brasil con vacas Nelore y Nelore x Angus tratadas con diferentes protocolos de sincronización del estro y la ovulación, han reportado que una mayor CC al inicio del protocolo fue asociada con un aumento en la tasa de preñez $(7,13,18)$. En vacas de primer parto Nelore y Nelore $x$ Angus Rojo sometidas a un protocolo de IATF basado en $\mathrm{P}_{4} / \mathrm{E}_{2}$ y destete temporal, Meneghetti y Vasconcelos (17) encontraron que, el incremento promedio en la tasa de concepción fue de $6 \%$ por cada aumento de 0.25 puntos en la CC (escala de 1 a 5).

Sá Filho et al (4) concluyeron que, los resultados contradictorios obtenidos acerca del efecto de la CC sobre la tasa de preñez en vacas Nelore de Brasil, pudieron deberse al estado reproductivo de las hembras (anestro o ciclicidad), el amamantamiento y la severidad del balance energético negativo. En la presente investigación, cabe anotar que, la gran mayoría de las vacas se encontraba en una CC $\geq 3.25(n=24)$. Esto podría explicar en parte, la falta de relación entre la CC y la tasa de preñez por IATF, la cual pudo deberse, probablemente, a un menor balance energético negativo de las vacas, lo cual les permitió mantener o recuperar rápidamente la CC después del parto, sin cambios agudos y/o importantes que pudieran afectar su desempeño reproductivo posparto.

Asimismo, el tratamiento con eCG potencialmente causa un mayor crecimiento folicular y proporciona un ambiente endocrino más adecuado durante el proestro (grandes concentraciones de $E_{2}$ en sangre) y el diestro (grandes concentraciones de $\mathrm{P}_{4}$ en sangre), el cual es favorable para la fertilidad (4). De esta forma, puede contribuir a aumentar las tasas de ovulación y las tasas de preñez en vacas (18), principalmente, cuando se encuentran en anestro, con baja CC (28) o ambos. Es probable que en la presente investigación, la eCG hubiera mejorado las tasas de ovulación y de preñez en las vacas con baja CC, razón por la cual al compararlas con las vacas de buena CC, no se logró establecer un efecto de la CC sobre la tasa de preñez por IATF.

En el presente estudio, la manifestación de estro no afectó la tasa de preñez por IATF. Contrario a ésto, en varias investigaciones hechas con novillas y vacas, tratadas con diferentes hormonas para sincronizar el estro y la ovulación, las hembras que manifestaron estro tuvieron una mayor tasa de preñez que aquellas que no lo mostraron $(3,7,14,29)$.

En esta investigación, se determinó que, a medida que se incrementó el diámetro del folículo mayor en el momento de la IATF, hubo una tendencia a aumentar la probabilidad de preñez por IATF. Resultados similares se han obtenido en estudios previos realizados con hembras bovinas tratadas con varios protocolos hormonales $(3,7,9,14,15,30,31)$. Sin embargo, en este experimento, no se estableció efecto de la categoría del diámetro del folículo mayor sobre la tasa de preñez por IATF. Contrariamente, en novillas Nelore, al clasificar los animales de acuerdo al diámetro del folículo mayor en el momento de la IATF (clase $1: \leq 8.5 \mathrm{~mm}$; clase 2: entre 8.5 y $10 \mathrm{~mm}$ y clase $3:>10 \mathrm{~mm}$ ), se encontró que la tasa de concepción incrementó con cada clase de tamaño del folículo (25). Asimismo, en búfalas, en el análisis por categorías del diámetro del folículo preovulatorio, la tasa de concepción aumentó con cada categoría folicular (32).

Las concentraciones plasmáticas de $E_{2}$ no influenciaron la tasa de preñez por IATF en el presente estudio. En vacas (14) y novillas de carne Bos taurus (19) se encontraron resultados similares. De forma opuesta, en vacas Holstein (30) y en búfalas (32), las concentraciones de $\mathrm{E}_{2}$ en plasma el día de la inseminación artificial influyeron positivamente sobre la preñez.

Bridges et al (24) concluyeron que, la madurez folicular no se pronostica de forma precisa por una sola característica, sino que, muy probablemente, sea producto de los efectos acumulativos de muchos factores, como la duración del proestro y la producción de $\mathrm{E}_{2}$ durante el mismo; el diámetro y la edad del folículo y la producción de $\mathrm{P}_{4}$ por el cuerpo lúteo resultante. En el presente trabajo, es posible que, las altas concentraciones de $\mathrm{E}_{2}$ en plasma $(23.99 \pm 0.96 \mathrm{pg} / \mathrm{ml})$ no fueran representativas de la madurez del folículo, por lo cual no se encontró relación de esta variable con la tasa de preñez por IATF. 
En las vacas del presente experimento, la tasa de preñez por monta natural en el ciclo estral siguiente al protocolo de IATF (20 días) fue de $36.8 \%$. Sá Filho et al (4) reportaron en vacas Nelore de primer parto, que el CIDR pero no la eCG, afectó la tasa de preñez al día 30 de la estación de reproducción (detección de estro dos veces al día después del retiro del CIDR, día 0 hasta el día 60). Esto se pudo deber a que la mayoría de los animales estaban en anestro, así que el tratamiento con CIDR combinado con destete temporal redujo el intervalo al estro y estimuló el inicio de ciclos estrales, mejorando el porcentaje de vacas exhibiendo estros durante la estación de reproducción. En vacas Bos indicus cruzadas en amamantamiento, la aplicación de eCG aumentó las concentraciones de $\mathrm{P}_{4}$ en plasma 12 días después de la terminación del tratamiento (2). Es posible que, algunos animales del presente estudio se encontraran en anestro $y$, por tanto, respondieron mejor en el ciclo estral siguiente, al reiniciar su ciclicidad ovárica por efecto del tratamiento con CIDR y eCG.

En Colombia existen inconvenientes para ejecutar proyectos científicos a nivel de campo, debido a la difícil consecución de ganaderos dispuestos a prestar sus animales para investigación, lo cual implica para ellos movilización de los animales y estrés por manejo, con la consecuente disminución de la producción. Asimismo, para el investigador es complicado encontrar grupos de animales homogéneos en cuanto a edad, número de partos, tiempo posparto, entre otras características, lo cual permitiría controlar de manera más confiable las variables que pueden afectar los resultados esperados.

Bajo las condiciones de la presente investigación, se puede concluir que, no hubo relación de la categoría de la CC, la categoría del diámetro del folículo mayor y las concentraciones de $\mathrm{E}_{2}$ con la manifestación de estro. El diámetro del folículo mayor y las concentraciones plasmáticas de $\mathrm{E}_{2}$ en el momento de la IATF, no mostraron correlación con la CC. No se encontró relación entre el diámetro del folículo mayor y las concentraciones plasmáticas de $E_{2}$. Se determinó una tendencia de una relación positiva entre el diámetro del folículo mayor y la tasa de preñez por IATF. Sin embargo, no se observó asociación de la CC, las concentraciones de $\mathrm{E}_{2}$ y la manifestación de estro con la tasa de preñez por IATF.

\section{Agradecimientos}

A la Vicerrectoría de Investigaciones y Postgrados de la Universidad de Caldas por la financiación del proyecto, a los propietarios y empleados de la Hacienda La Indiana por la colaboración en la realización del estudio, al Dr. Diego Villamizar Ramírez y a Miryam Vélez Marín por el apoyo en el trabajo de campo y al Dr. Henry Mesa Echeverri, por la asesoría en el diseño experimental y en el análisis estadístico.

\section{REFERENCIAS}

1. Baruselli PS, Gimenes LU, Sales JNS. Fisiologia reprodutiva de fêmeas taurinas e zebuínas. Rev Bras Reprod Anim 2007; 31(2):205-211.

2. Baruselli PS, Reis EL, Marques MO, Nasser LF, Bo GA. The use of hormonal treatments to improve reproductive performance of anestrous beef cattle in tropical climates. Anim Reprod Sci 2004; 83:479-486.

3. Peres RFG, Claro Júnior I, Sá Filho OG, Nogueira GP, Vasconcelos JLM. Strategies to improve fertility in Bos indicus postpubertal heifers and nonlactating cows submitted to fixed-time artificial insemination. Theriogenology 2009; 72:681-689.
4. Sá Filho OG, Dias CC, Lamb GC, Vasconcelos JLM. Progesterone-based estrous synchronization protocols in non-suckled and suckled primiparous Bos indicus beef cows. Anim Reprod Sci 2010; 119:9-16.

5. Bó GA, Baruselli PS, Martínez MF. Pattern and manipulation of follicular development in Bos indicus cattle. Anim Reprod Sci 2003; 78:307-326.

6. Vasconcelos JLM, Sartori R, Oliveira $H N$, Guenther JG, Wiltbank MC. Reduction in size of the ovulatory follicle reduces subsequent luteal size and pregnancy rate. Theriogenology 2001; 56:307-314. 
7. Sá Filho MF, Crespilho AM, Santos JEP, Perry GA, Baruselli OS. Ovarian follicle diameter at timed insemination and estrous response influence likelihood of ovulation and pregnancy after estrous synchronization with progesterone or progestin-based protocols in suckled Bos indicus cows. Anim Reprod Sci 2010b; 120:23-30.

8. Federación Colombiana de Ganaderos FEDEGAN. Plan Estratégico de la Ganadería Colombiana 2019. $1^{\text {a }}$ ed. Bogotá D.C., Colombia: Sanmartín Obregón \& Cía; 2006.

9. Meneghetti M, Sá Filho OG, Peres RFG, Lamb GC, Vasconcelos JLM. Fixed-time artificial insemination with estradiol and progesterone for Bos indicus cows I: basis for development of protocols. Theriogenology 2009; 72:179-189.

10. Instituto de Hidrología, Meteorología y Estudios Ambientales - IDEAM. Atlas Climatológico de Colombia. Segunda parte: distribución espacio-temporal de las variables del clima. $1^{\text {a }}$ ed. Bogotá D.C., Colombia: Imprenta Nacional de Colombia; 2005, p. 21- 108.

11. Maciel ABB. Proposta de avaliação da condição corporal em vacas Holandesas e Nelore [Tesis de Maestría]. Botucatu, Brasil: Universidade Estadual Paulista - UNESP; 2006.

12. SAS Institute Inc. $®:$ Guide for Personal computer [programa de ordenador]. Versión 5.0. Cary (NC): SAS Institute IC.; 1985.

13. Vasconcelos JLM, Sá Filho OG, Perez GC, Silva AT. Intravaginal progesterone device and/or temporary weaning on reproductive performance of anestrous crossbred Angus $x$ Nelore cows. Anim Reprod Sci 2009; 111:302-311.

14. Perry GA, Smith MF, Lucy MC, Green JA, Parks TE, MacNeil MD et al. Relationship between follicle size at insemination and pregnancy success. PNAS 2005; 102(14):5268-5273.

15. Sá Filho OG, Meneghetti M, Peres RFG, Lamb GC, Vasconcelos JLM. Fixed-time artificial insemination with estradiol and progesterone for Bos indicus cows II: strategies and factors affecting fertility. Theriogenology 2009a; 72:210-218.
16. Lents CA, White FJ, Ciccioli NH, Wettemann RP, Spicer LJ, Lalman DL. Effects of body condition score at parturition and postpartum protein supplementation on estrous behavior and size of the dominant follicle in beef cows. J Anim Sci 2008; $86: 2549-2556$.

17. Meneghetti M, Vasconcelos JLM. Mês de parição, condição corporal e resposta ao protocolo de inseminação artificial em tempo fixo em vacas de corte primíparas. Arq Bras Med Vet Zootec 2008; 60(4):786-793.

18. Sá Filho MF, Ayres H, Ferreira RM, Marques MO, Reis EL, Silva RCP et al. Equine chorionic gonadotropin and gonadotropinreleasing hormone enhance fertility in a norgestomet-based, timed artificial insemination protocol in suckled Nelore (Bos indicus) cows. Theriogenology 2010a; 73:651-658.

19. Lynch CO, Kenny DA, Childs S, Diskin MG. The relationship between periovulatory endocrine and follicular activity on corpus luteum size, function, and subsequent embryo survival. Theriogenology 2010; 73:190-198.

20. Sá Filho OG, Thatcher WW, Vasconcelos JLM. Effect of progesterone and/or estradiol treatments prior to induction of ovulation on subsequent luteal lifespan in anestrous Nelore cows. Anim Reprod Sci 2009b; 112:95-106.

21. Atkins JA, Smith MF, Wells KJ, Geary TW. Factors affecting preovulatory follicle diameter and ovulation rate after gonadotropin-releasing hormone in postpartum beef cows. Part I: Cycling cows. J Anim Sci 2010; 88:2300-2310.

22. Sá Filho MF, Torres-Júnior JRS, Penteado L, Gimenes LU, Ferreira RM, Ayres $\mathrm{H}$, et al. Equine chorionic gonadotropin improves the efficacy of a progestin-based fixed-time artificial insemination protocol in Nelore (Bos indicus) heifers. Anim Reprod Sci 2010c; 118:182-187.

23. Colazo MG, Kastelic JP, Mapletoft RJ. Effects of estradiol cypionate (ECP) on ovarian follicular dynamics, synchrony of ovulation, and fertility in CIDR-based, fixed-time AI programs in beef heifers. Theriogenology 2003; 60:855-865. 
24. Bridges GA, Mussard ML, Burke CR, Day ML. Influence of the length of proestrus on fertility and endocrine function in female cattle. Anim Reprod Sci 2010; 117:208-215.

25. Dias CC, Wechsler FS, Day ML, Vasconcelos JLM. Progesterone concentrations, exogenous equine chorionic gonadotropin, and timing of prostaglandin $F_{2 a}$ treatment affect fertility in postpuberal Nelore heifers. Theriogenology 2009; 72:378-385.

26. Pinheiro VG, Souza AF, Pegorer MF, Satrapa RA, Ereno RL, Trinca LA et al. Effects of temporary calf removal and eCG on pregnancy rates to timed-insemination in progesterone-treated postpartum Nellore cows. Theriogenology 2009; 71:519-524.

27. Figueira Pegorer $M$, Ereno RL, Satrapa RA, Pinheiro VG, Trinca LA, Barros CM. Neither plasma progesterone concentrations nor exogenous eCG affects rates of ovulation or pregnancy in fixed-time artificial insemination (FTAI) protocols for puberal Nellore heifers. Theriogenology 2011; 75:17-23.

28. Sales JNS, Crepaldi GA, Girotto RW, Souza $A H$, Baruselli PS. Fixed-time AI protocols replacing eCG with a single dose of $\mathrm{FSH}$ were less effective in stimulating follicular growth, ovulation, and fertility in suckledanestrus Nelore beef cows. Anim Reprod Sci 2011; 124:12-18.
29. Sá Filho MF, Santos JEP, Ferreira RM, Sales JNS, Baruselli PS. Importance of estrus on pregnancy per insemination in suckled Bos indicus cows submitted to estradiol/ progesterone-based timed insemination protocols. Theriogenology 2011; 76:455-463.

30. Lopes AS, Butler ST, Gilbert RO, Butler WR. Relationship of pre-ovulatory follicle size, estradiol concentrations and season to pregnancy outcome in dairy cows. Anim Reprod Sci 2007; 99:34-43.

31. Cavestany D, Crespi D, Fernández A. Oestrus synchronisation and fixed time artificial insemination in beef heifers. Anim Prod Sci 2010; 50:670-674.

32. Pandey AK, Dhaliwal GS, Ghuman SPS, Agarwal SK. Impact of pre-ovulatory follicle diameter on plasma estradiol, subsequent luteal profiles and conception rate in buffalo (Bubalus bubalis). Anim Reprod Sci 2011; 123:169-174. 